\title{
INTERNATIONAL JOURNAL OF \\ PRACTICAL THEOLOGY
}

\section{EDITORS}

Pamela Couture, Toronto, $\mathrm{ON}$

Bernd Schröder, Göttingen

IN COOPERATION WITH

Nadine Bowers du Toit, Stellenbosch

Jaco Dreyer, Pretoria

Elaine Graham, Chester

Wilhelm Gräb, Berlin

Christian Grethlein, Münster

Thomas Hastings, New Haven, CT

Solange Lefebvre, Montreal, QC

Norbert Mette, Dortmund

Bonnie J. Miller-McLemore, Nashville, TN

Mary Elizabeth Moore, Boston, MA

James Nieman, Chicago, IL

Richard R. Osmer, Princeton, NJ

Nancy Ramsay, Fort Worth, TX

Robert Schreiter, Chicago, IL

Friedrich Schweitzer, Tübingen

Henrik Simojoki, Bamberg

Joon Kwan Un, Seoul

Johannes A. van der Ven, Nijmegen

Claire Wolfteich, Boston, MA

\section{DE GRUYTER}


ABSTRACTED/INDEXED IN ATLA Religion Database · Baidu Scholar · Base d'information bibliographique en patristique / Bibliographic Information Base in Patristics (BIBP) · Clarivate Analytics: Emerging Sources Citation Index; Web of Science · CNKI Scholar (China National Knowledge Infrastructure) CNPIEC - De Gruyter: IBR (International Bibliography of Reviews of Scholarly Literature in the Humanities and Social Sciences); IBZ (International Bibliography of Periodical Literature in the Humanities and Social Sciences) · EBSCO (relevant databases) · EBSCO Discovery Service · Elsevier: SCOPUS · ERIH PLUS (European Reference Index for the Humanities and Social Sciences) · Gale/Cengage · Genamics JournalSeek · Google Scholar · Index Theologicus · J-Gate · JournalGuide · JournalTOCs · KESLI-NDSL (Korean National Discovery for Science Leaders) · Microsoft Academic · Naviga (Softweco) · Primo Central (ExLibris) · ProQuest (relevant databases) · Publons · ReadCube · Religious and Theological Abstracts · RKE Online (Religionspädagogik, Kirchliche Bildungsarbeit, Erziehungswissenschaft) - SCImago (SJR) - Sherpa/RoMEO · Summon (Serials Solutions/ProQuest) - TDNet · Ulrich`s Periodicals Directory/ ulrichsweb $\cdot$ WanFang Data $\cdot$ WorldCat (OCLC)

ISSN 1430-6921 · e-ISSN 1612-9768

All information regarding notes for contributors, subscriptions, Open access, back volumes and orders is available online at www.degruyter.com/ijpt.

EDITORS Prof. Dr. Pamela Couture, Ph.D., Emmanuel College of Victoria University in the University of Toronto, 75 Queen's Park Crescent, Toronto, ON M5S 1K7, Canada,

E-mail: pamela.couture@utoronto.ca

Prof. Dr. Bernd Schröder, Georg-August-Universität Göttingen, Theologische Fakultät, Platz der Göttinger Sieben 2, 37073 Göttingen, Germany, E-mail: bernd.schroeder@theologie.uni-goettingen.de

BOOK REVIEW EDITORS Prof. Dr. Nadine Bowers du Toit, Stellenbosch University, 171 Dorp St., Stellenbosch 7600, Republic of South Africa, Email: nbowers@sun.ac.za

Prof. Dr. Nancy Ramsay, Brite Divinity School, TCU Box 298130, Fort Worth, TX 76129 USA, Email: n.ramsay@tcu.edu

Prof. Dr. Henrik Simojoki, Otto-Friedrich-Universität Bamberg, Markusplatz 3, 96045 Bamberg,

Germany, E-mail: henrik.simojoki@uni-bamberg.de

EDITORSHIP Moritz Emmelmann, Georg-August-Universität Göttingen, Theologische Fakultät, Platz der Göttinger Sieben 2, 37073 Göttingen, Germany,

E-mail: moritz.emmelmann@theologie.uni-goettingen.de

Prof. Dr. Stephen Simmons, retired, Moravian Theological Seminary, 60 West Locust Street, Bethlehem, PA 18018 USA, Email: simmonss@ moravian.edu

JOURNAL MANAGER Sabine von Wittke-Holweg, De Gruyter, Genthiner Straße 13, 10785 Berlin, Germany, Tel.: +49 (0)30 260 05-334, Fax: +49 (0)30 260 05-250,

E-mail: sabine.von.wittke@degruyter.com

RESPONSIBLE FOR ADVERTISEMENTS Claudia Neumann, De Gruyter, Genthiner Straße 13, 10785 Berlin, Germany, Tel.: +49 (0)30 260 05-226, Fax: +49 (0)30 260 05-264,

E-mail: anzeigen@degruyter.com

(C) 2018 Walter de Gruyter GmbH, Berlin/Boston

TYPESETTING jürgen ulrich typosatz, Nördlingen

PRINTING Franz X. Stückle Druck und Verlag e.K., Ettenheim

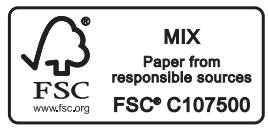




\section{Contents}

Bernd Schröder

Editorial — 1

\section{Research Articles}

Tobias Brandner

A Case Study on Conversion in the Context of Prison in Hong Kong: Experiences, Narratives, and Transformations - 3

Aino-Elina Kilpeläinen und Marie Vejrup Nielsen

Teaching Rituals: New Church Activities and Religious Education - 23

Hans Malmström

Appraisal, Preaching and the Religious Other: The Rhetorical Appropriation of Interreligious Positions in Sermonic Discourse - $\mathbf{4 0}$

Hee-Kyu Heidi Park

Postcolonializing Practical Theological Methodology as Cartography of Boundary Dynamics — 58

Jos de Kock und Bård E. Hallesby Norheim

Youth Ministry Research and the Empirical -69

Tone Stangeland Kaufman und Jonas Ideström

Why Matter Matters in Theological Action Research: Attending to the Voices of Tradition -84

\section{International Report}

Roy Lazar A.

A Theory of Religious Praxis in India. Introduction to Practical Theology in India - Part II -103 


\section{Book Reviews}

Darren Cronshaw

Miroslav Volf, Flourishing: Why We Need Religion in a Globalized World - 129

Courtney T. Goto

Evelyn L. Parker, Between Sisters: Emancipatory Hope out of Tragic Relationships - 131

Mary Clark Moschella

Pamela Couture, We Are Not All Victims: Local Peacebuilding in the Democratic Republic of Congo 135

Mai-Anh Le Tran

Joyce Ann Mercer and Bonnie J. Miller-McLemore, eds., Conundrums in Practical Theology 139

\section{Andreas Kubik}

Emilia Handke: Religiöse Jugendfeiern „zwischen Kirche und anderer Welt“. Eine historische, systematische und empirische Studie über kirchlich (mit)verantwortete Alternativen zur Jugendweihe - 143

Gerald Kretzschmar

Jantine Nierop: Eine Gemeinde, mehrere PfarrerInnen. Reflexionen auf das mehrstellige Pfarramt aus historischer, empirischer und akteurtheoretischer Perspektive - 148 